\title{
SIRT5 regulates pancreatic $\beta$-cell proliferation and insulin secretion in type 2 diabetes
}

\author{
YONGMEI MA and XIAOQIANG FEI \\ Department of Endocrinology, Taizhou People's Hospital, Taizhou, Jiangsu 225300, P.R. China
}

Received July 30, 2017; Accepted October 27, 2017

DOI: $10.3892 /$ etm.2018.6301

\begin{abstract}
Impaired insulin secretion and insulin resistance are the primary characteristics of type 2 diabetes (T2D). However, the mechanisms underlying insulin secretion failure have yet to be elucidated. The present study demonstrated that sirtuin 5 (SIRT5) is upregulated in patients with T2D and in pancreatic $\beta$-cell lines. It was also revealed that elevated SIRT5 expression is positively associated with age and blood glucose levels, and negatively associated with pancreatic and duodenal homeobox 1 (PDX1) expression. Colony formation and Cell Counting Kit-8 assays demonstrated that SIRT5 suppressed the proliferation of pancreatic $\beta$-cells in vitro. In addition, downregulation of SIRT5 promoted the secretion of insulin. Additionally, SIRT5 ectopic expression downregulated the expression of PDX1 and the inhibition of SIRT5 upregulated PDX1 expression. Chromatin immunoprecipitation assay analysis demonstrated that SIRT5 may regulate the transcription of PDX1 via H4K16 deacetylation. In conclusion, the results of the present study indicate that SIRT5 may serve an important role in the pathogenesis of $\mathrm{T} 2 \mathrm{D}$, and may present a novel therapeutic target for the treatment of patients with T2D.
\end{abstract}

\section{Introduction}

Diabetes mellitus is a chronic disease that affects $~ 500$ million people worldwide (1). Functional $\beta$-cell apoptosis and dysfunctional insulin secretion are the two primary mechanisms that are thought to be involved in the pathogenesis of type 2 diabetes (T2D) (2-6). Collectively, these mechanisms result in the inability to compensate for insulin resistance. Therefore, enhancing $\beta$-cell proliferation and increasing insulin secretion may be novel strategies for the treatment of diabetes.

Sirtuins (SIRTs) are proteins that possess nicotinamide-adenine dinucleotide-dependent deacetylase activity

Correspondence to: Dr Xiaoqiang Fei, Department of Endocrinology, Taizhou People's Hospital, 399 Hailing Road, Hailing, Taizhou, Jiangsu 225300, P.R. China

E-mail: oqy1kbm@163.com

Key words: sirtuin 5, proliferation, insulin secretion, pancreatic and duodenal homeobox 1 , type 2 diabetes and belong to the histone deacetylase (HDAC) family, which consist of seven different subtypes in mammals (SIRT1-7) (7). SIRTs were originally identified to be involved in lifespan prolongation; however, research has revealed that they serve major roles in a number of additional biological processes, including cell metabolism, aging and tumorigenesis (7).

Although SIRT5 possesses weak deacetylase activity, it catalyzes the modification of acidic lysine residues by glutarylation, succinylation and malonylation (8-12). Previous studies have identified thousands of potential SIRT5 targets, including metabolic enzymes involved in ketone body synthesis, the tricarboxylic acid cycle, amino acid catabolism, fatty-acid $\beta$-oxidation, glycolysis and ATP synthesis (13-15). However, the functional role of SIRT5 in T2D remains unknown. SIRT5 regulates the expression of various metabolic enzymes and may also serve a key function in T2D. The present study aimed to determine the roles and underlying mechanism of SIRT5 in T2D.

\section{Materials and methods}

Cell culture. MIN6 and INS-1 cells were purchased from the American Type Culture Collection (Manassas, VA, USA) and cultured in low-glucose Dulbecco's modified Eagle's medium (5 mmol/1 glucose; HyClone; GE Healthcare Life Sciences, Logan, UT, USA), supplemented with $100 \mathrm{U} / \mathrm{ml}$ penicillin, $0.1 \mathrm{mg} / \mathrm{ml}$ streptomycin (Invitrogen; Thermo Fisher Scientific, Inc., Waltham, MA, USA), $5.5 \mathrm{mM}$ 2-mercaptoethanol and $10 \%$ horse serum (Invitrogen; Thermo Fisher Scientific, Inc.) at $37^{\circ} \mathrm{C}$ with $5 \% \mathrm{CO}_{2}$.

Blood sample collection. The present study was approved by the Ethics Committee of Taizhou People's Hospital (Taizhou, China), and written informed consent was provided from patients and healthy volunteers prior to enrolment. Blood samples were collected from 130 patients, including 65 patients with T2D patients (that had not yet received treatment) and 65 healthy volunteers that were admitted to Taizhou People's Hospital between 2015 to 2016, and stored at $-80^{\circ} \mathrm{C}$ prior to use. Healthy control subjects were recruited from age- and gender-matched healthy blood donors. Patients were diagnosed according to the criteria of the American Diabetes Association (16). T2D patients contained 30 males and 36 females, with age range of 11-87 years and an average age of $40 \pm 0.55$ years. Blood glucose levels were determined 
using ACCU-CHEK (Roche Diagnostics GmbH, Mannheim, Germany).

Plasmid and transfection. pcDNA3.1-SIRT5 and pcDNA3.1-pancreatic and duodenal homeobox 1 (PDX1) were purchased from Vigene Biosciences (Rockville, MD, USA). The inactive form of the SIRT5 deacetylase (T69A) was mutated using a Fast Mutagenesis System (Beijing Transgen Biotech Co., Ltd., Beijing, China) according to the manufacturer's protocol. RNAi-resistant SIRT5 (SIRT5R) was mutated using Fast Mutagenesis System according to the sequence of SIRT5 siRNA. For overexpressing SIRT5, a total of $2.5 \mu \mathrm{g}$ vector (pcDNA3.1) and indicated plasmid were transfected into MIN6 and INS-1 cells using lipo 2000 (Thermo Fisher Scientific Inc.). Following transfection for 48 h, cells were collected and used for subsequent experiments.

RNA interference. A total of $4 \times 10^{5}$ INS-1 or MIN6 cells were transfected with $50 \mathrm{nM}$ SIRT5 small interfering RNA (siSIRT5) or scrambled siRNA (SCR, Sigma-Aldrich, St. Louis, MO, USA) using the DharmaFECT 1 kit (Thermo Fisher Scientific Inc.) according to the manufacturers' protocol. Following transfection for $48 \mathrm{~h}$, the expression of SIRT5 was determined using reverse transcription quantitative polymerase chain reaction (RT-qPCR) and western blotting. Each experiment was repeated three times. SIRT5 siRNA was synthesized form Sigma-Aldrich (Darmstadt, Germany). The sequences for SIRT5 were as follows: siRNA\#1, GAGAAU UACAAGAGUCCAAUU and siRNA\#2, GGAGAUCCA UGGUAGCUUAUU.

$R T-q P C R$. Total RNA from blood samples and INS-1 or MIN6 cells was extracted using the TRIzol reagent (Invitrogen; Thermo Fisher Scientific, Inc.) according to the manufacturer's protocol. A total of $2 \mu \mathrm{g}$ RNA was reverse transcribed into cDNA using the EasyScript First-Strand cDNA Synthesis SuperMix kit (TransGen Biotech Co., Ltd., Beijing, China) according to the manufacturer's protocol. A SYBR green Mix (Roche Diagnostics $\mathrm{GmbH}$ ) was used to perform RT-qPCR assay. The thermal cycling parameters were as follows: 33 cycles of denaturation at $95^{\circ} \mathrm{C}$ for $4 \mathrm{~min}$, annealing at $55^{\circ} \mathrm{C}$ for $30 \mathrm{sec}$ followed by extension at $72^{\circ} \mathrm{C}$ for $8 \mathrm{~min}$. The following primers were used: SIRT5, forward, 5'-AAATAA CTAAAGCCCGCCTC-3', and reverse, 5'-CCACTTTCT GCACTAACACC-3'; PDX1, forward, 5'-CCTTTCCCATGG ATGAAGTC-3', and reverse, 5'-GTGAGATGTACTTGTTGA ATAGGA-3'; glyceraldehyde-3-phosphate dehydrogenase (GAPDH), forward, 5'-ATGATGACATCAAGAAGGTGG-3', and reverse, 5'-TTGTCATACCAGGAAATGAGC-3'. The relative mRNA expression was normalized to that of GAPDH and quantified using the $2^{-\Delta \Delta C t}$ method (17). Each experiment was repeated three times.

Western blotting. Cells were harvested and lysed in radioimmunoprecipitation assay (RIPA, Beyotime Institute of Biotechnology, Jiangsu, China) buffer, and the protein concentration of sample extracts was determined using a BCA protein assay kit (Pierce; Thermo Fisher Scientific, Inc.). A total of $45 \mu \mathrm{g}$ protein was loaded per lane and electrophoresed by $8 \%$ SDS-PAGE. Samples were then transferred to a polyvinylidene fluoride membrane. Membranes were blocked in 5\% non-fat milk at room temperature for $1 \mathrm{~h}$. Membranes were then incubated with primary antibodies against SIRT5 (1:1,000; cat. no. ab108968), PDX1 (1:1,000; cat. no. ab134150) or $\beta$-actin $\left(1: 7,000\right.$; cat. no. ab8226) overnight at $4^{\circ} \mathrm{C}$ (All Abcam, Cambridge, UK). Following washing three times with tris-buffered saline Tween 20, membranes were incubated with secondary horseradish peroxidase-conjugated antibodies (goat anti mouse, 1:3,000; ab6789; Abcam, Cambridge, UK; goat anti rabbit, 1:5,000, ab205718; Abcam, Cambridge, UK) at room temperature for $1 \mathrm{~h}$. Specific protein bands were visualized using a BeyoECL Plus ECL kit (Beyotime Institute of Biotechnology). Image J software (version 1.48; National Institutes of Health, Besthesda, MD, USA) was used for densitometry.

CCK-8 assay. A CCK-8 assay was used to investigate the effect of SIRT5 expression on cell proliferation. INS-1 and MIN6 cells were transfected with $2.5 \mu \mathrm{g}$ pcDNA 3.1 vector, SIRT5 plasmid (Vigene Biosciences, Rockville, MD, USA) or SIRT5 and PDX1 plasmid, $50 \mathrm{nM}$ scramble siRNA (SCR) or SIRT5 siRNA (siSIRT5, Sigma-Aldrich, St. Louis, MO, USA), respectively. Following transfection for $48 \mathrm{~h}$, a total of $3 \times 10^{3}$ cells were seeded into 96 -well plates with $200 \mu$ l low-glucose Dulbecco's Modified Eagle's medium $(5 \mathrm{mmol} / \mathrm{l}$ glucose; HyClone; GE Healthcare Life Sciences) supplemented with $10 \%$ horse serum (Invitrogen; Thermo Fisher Scientific, Inc.). Subsequently, $20 \mu$ l CCK- 8 reagent (Beyotime Institute of Biotechnology) was added to each well and cells were incubated for a further $1 \mathrm{~h}$ at $37^{\circ} \mathrm{C}$. Finally, the absorbance at $450 \mathrm{~nm}$ was read using a microplate reader (BioTek Instruments Inc., Winooski, VT, USA). Each experiment was repeated three times.

Colony formation assay. SIRT5 was overexpressed or knocked down in INS-1 and MIN6 cells. Following transfection for $48 \mathrm{~h}, \sim 2 \times 10^{4}$ transfected INS-1 and MIN6 cells were seeded into 6-well plates. Cells were cultured with serum-free low-glucose Dulbecco's modified Eagle's medium for 14 days. Subsequently, cells were fixed with methanol at room temperature for $10 \mathrm{~min}$ and stained with $0.5 \%$ crystal violet at room temperature for $10 \mathrm{~min}$. The colonies (cells $>50$ ) were counted under a light microscope (magnification, 20x). Each experiment was repeated three times. The Image J software (v1.04; National Institutes of Health, Bethesda, MD, USA) was used to assess colony formation

ChIP and qChIP assay. A ChIP assay was performed with $2.5 \mu \mathrm{g}$ anti-H4K16ac (cat. no. ab109463; Abcam) and goat anti-rabbit IgG (cat. no. ab171870; Abcam) using a ChIP assay kit (Beyotime Institute of Biotechnology) according to the manufacturer's protocol. The antibody-bound DNA was detected in $2 \%$ agarose gel. Subsequently, antibody-bound DNA was used to perform qChIP. SYBR green Mix (Roche Diagnostics $\mathrm{GmbH}$ ) was used to perform qPCR. The thermocycling conditions were as follows: 30 cycles of denaturation at $95^{\circ} \mathrm{C}$ for $5 \mathrm{~min}$, annealing at $56^{\circ} \mathrm{C}$ for $30 \mathrm{sec}$ followed by extension at $72^{\circ} \mathrm{C}$ for $5 \mathrm{~min}$. The following primers were used: PDX1, forward, 5'-AGGGTCTCATTCTGTCGTTC-3'; PDX1, reverse, 5'-GCCTGTAATCCCAGCTACTC-3'. The fold of 

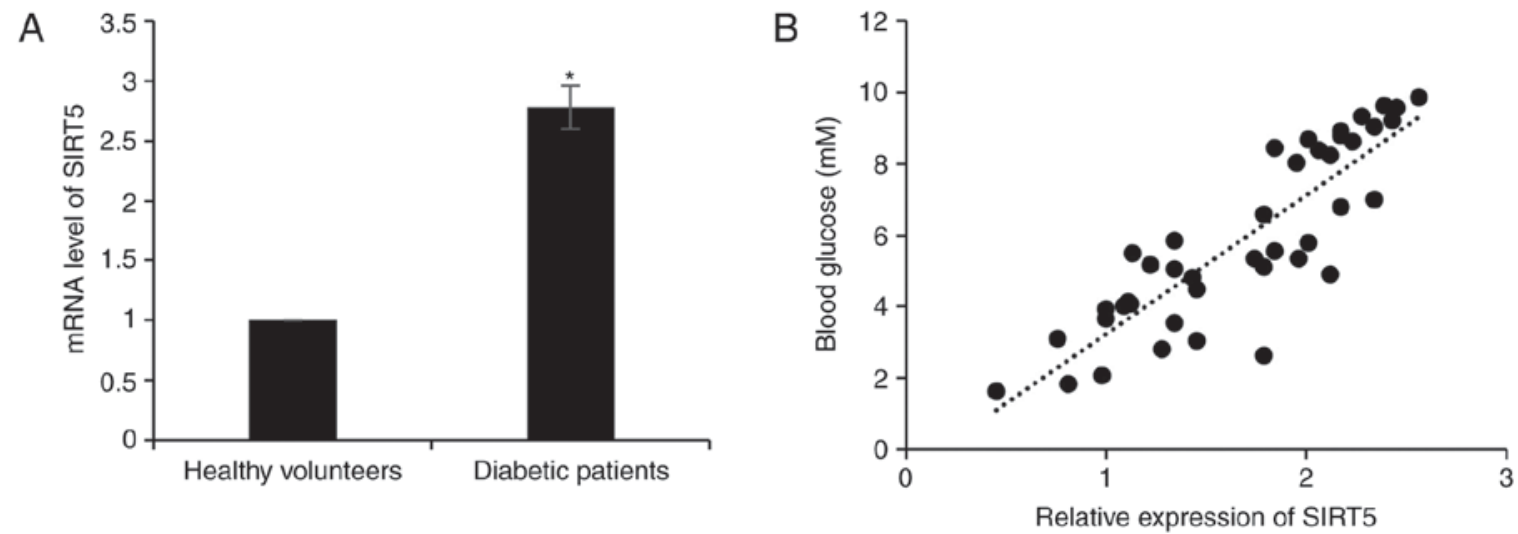

Figure 1. SIRT5 expression is significantly upregulated in patients with type 2 diabetes. (A) mRNA levels of SIRT5 in diabetic patients and healthy volunteers were detected using reverse transcription quantitative polymerase chain reaction analysis. Each experiment was repeated three times. (B) Pearson's correlation analysis was performed to determine the association between the SIRT5 expression and blood glucose levels in patients with type 2 diabetes. The results are presented as the mean \pm standard deviation. " $\mathrm{P}<0.05$ vs. healthy volunteers. SIRT5, sirtuin 5.

enrichment was normalized to that of $\mathrm{IgG}$ and quantified using the $2^{-\Delta \Delta \mathrm{Cq}}$ method (17). Each experiment was repeated three times.

Insulin secretion and insulin content measurements. A total of $\sim 4 \times 10^{5}$ cells were seeded into a 24 -well plate and transfected with a pcDNA 3.1 vector, SIRT5 plasmid or SIRT5 and PDX1 plasmid, scramble siRNA (SCR) or SIRT5 siRNA (siSIRT5). Following transfection for $24 \mathrm{~h}$, MIN6 and INS-1 cells were also incubated with 1 or $20 \mathrm{mM}$ glucose for $90 \mathrm{~min}$ and the rate of insulin secretion was detected in each group. Cells were washed with a secretion assay buffer [sub arachnoid block (SAB); 20 mM HEPES (pH 7.2), $2.5 \mathrm{mM} \mathrm{CaCl}_{2}, 1.16 \mathrm{mM}$ $\mathrm{MgSO}_{4}, 1.2 \mathrm{mM} \mathrm{KH} \mathrm{PO}_{4}, 4.7 \mathrm{mM} \mathrm{KCl}, 25.5 \mathrm{mM} \mathrm{NaHCO}$, $114 \mathrm{mM} \mathrm{NaCl}$ and $0.2 \%$ bovine serum albumin (Invitrogen; Thermo Fisher Scientific, Inc.) with $2.8 \mathrm{mM}$ glucose three times. Subsequently, cells were incubated with $1.5 \mathrm{ml} \mathrm{SAB}$ at $37^{\circ} \mathrm{C}$ and $5 \% \mathrm{CO}_{2}$ for $2 \mathrm{~h}$. Immediately following incubation, insulin levels were analyzed using the Coat-A-Count insulin RIA kit (Diagnostic Products Corporation, Los Angeles, CA, USA)] according to the manufacturers' protocol. Cells were lysed with RIPA buffer [1\% NP-40/Triton X, $0.5 \%$ sodium deoxycholate, $0.1 \%$ SDS, $150 \mathrm{mM} \mathrm{NaCl}, 50 \mathrm{mM}$ Tris $\mathrm{HCl}$ (pH 8.0), $50 \mathrm{mM} \mathrm{NaF}$ and $2 \mathrm{mM}$ EDTA]. The cell lysates were centrifuged at $1 \times 10^{4} \mathrm{~g}$ for $5 \mathrm{~min}$ at $4^{\circ} \mathrm{C}$, and shaken on ice for $30 \mathrm{~min}$. The supernatant was collected and used for the analysis of insulin content. Total protein concentration was measured using a BCA Protein assay kit (Pierce; Thermo Fisher Scientific, Inc.) according to the manufacturer's protocol. The insulin content of each group was normalized to protein concentration. Each experiment was repeated three times.

Incubation of cells with valproic acid (VPA). INS-1 cells were transfected with vector (pcDNA3.1) or SIRT5 plasmid and cultured with or without $20 \mathrm{mM}$ glucose. A total of $0.1,0.5$ or $1 \mathrm{mM}$ VPA (cat. no. S1168; Selleck Chemicals, Shanghai, China) was added to cells immediately following transfection. The low-glucose Dulbecco's modified Eagle's medium ( $5 \mathrm{mmol} / \mathrm{l}$ glucose) supplemented with $10 \%$ horse serum was subsequently removed and VPA-containing medium was refreshed 1 day following transfection. Insulin secretion tests were also performed at $48 \mathrm{~h}$ following transfection and treatment with VPA. Each experiment was repeated three times.

Statistical analysis. Each experiment was repeated three times and the results are expressed as the mean \pm standard deviation. SPSS 18.0 software (SPSS, Inc., Chicago, IL, USA) was used for statistical analysis. The Student's t-test was used to analyze the differences between two groups and a one-way analysis of variance followed by a Tukey's post hoc test, was used to analyze differences among multiple groups. Associations between SIRT5 expression and blood glucose levels were determined using the Pearson's correlation coefficient. $\mathrm{P}<0.05$ was considered to indicate a statistically significant difference.

\section{Results}

Expression of SIRT5 is markedly upregulated in patients with $T 2 D$. In order to investigate the role of SIRT5 in diabetes, the expression of SIRT5 in plasma samples obtained from 65 patients with T2D and 65 healthy volunteers was assessed. The result of RT-qPCR analysis revealed that SIRT5 mRNA levels were significantly upregulated in patients with diabetes compared with healthy volunteers (Fig. 1A). It was also determined that the expression of SIRT5 was positively correlated with blood glucose levels ( $r=0.87$; Fig. 1B). In addition, the association between patient clinicopathological features and SIRT5 expression was analyzed, and it was revealed that a high SIRT5 expression was significantly associated with age, but not with gender (Table I).

SIRT5 suppresses insulin secretion and the proliferation of pancreatic $\beta$-cell lines. To elucidate the function of SIRT5 in diabetes, a pcDNA3.1-SIRT5 plasmid was constructed and SIRT5 was overexpressed or knocked down in MIN6 and INS-1 pancreatic $\beta$-cell lines. Following a 48 h transfection, the expression of SIRT5 was determined by RT-qPCR and western blotting analyses. SIRT5 was significantly upregulated and downregulated in MIN6 and INS-1 cells transfected with the pcDNA3.1-SIRT5 plasmid and siSIRT5, respectively, 
Table I. Association between SIRT5 expression and the clinicopathologic features of 65 patients with type 2 diabetes.

SIRT5 protein expression

\begin{tabular}{|c|c|c|c|c|}
\hline & & & & \\
\hline Characteristic & Number & Low $(n=23)$ & $\operatorname{High}(n=42)$ & P-value \\
\hline Age & & & & 0.011 \\
\hline$<40$ & 26 & 14 & 12 & \\
\hline$\geq 0$ & 39 & 9 & 30 & \\
\hline Sex & & & & 0.749 \\
\hline Male & 30 & 10 & 20 & \\
\hline Female & 35 & 13 & 22 & \\
\hline Blood glucose level & & & & $<0.001$ \\
\hline$<7.0 \mathrm{mM}$ & 25 & 15 & 10 & \\
\hline$>7.0 \mathrm{mM}$ & 40 & 8 & 32 & \\
\hline PDX1 expression & & & & $<0.001$ \\
\hline Low & 38 & 7 & 31 & \\
\hline High & 27 & 16 & 11 & \\
\hline
\end{tabular}

Data are presented as the mean \pm standard deviation. PDX1, pancreatic and duodenal homeobox 1 .

when compared with their respective controls (Fig. 2A and B). siSIRT5\#2 was demonstrated to be more efficient at repressing SIRT5 than siSIRT5\#1, and was therefore utilized in subsequent experiments. A colony formation assay was performed to assess the effect of SIRT5 expression on the proliferation of pancreatic $\beta$-cell lines. The results demonstrated that ectopic SIRT5 expression significantly suppressed MIN6 and INS-1 cell colony formation when compared with controls, whereas inhibition of SIRT5 expression had the opposite effect (Fig. 2C). In addition, the results of the CCK-8 assay indicated that upregulation of SIRT5 significantly inhibited MIN6 and INS-1 cell proliferation when compared with controls, whereas SIRT5 inhibition demonstrated the opposite effect (Fig. 2D). It was also demonstrated that overexpression of SIRT5 significantly decreased the level of insulin secretion following exposure to glucose when compared with controls, whereas inhibition of SIRT5 expression significantly increased insulin secretion (Fig. 2E). Therefore, the results demonstrated that SIRT5 downregulation facilitates insulin secretion and the proliferation of pancreatic $\beta$-cell lines.

PDX1 is transcriptionally regulated by SIRT5. As a HDAC enzyme, SIRT5 has been demonstrated to regulate the expression of genes (7). The protein PDX1 serves a number of key roles in the pathogenesis of diabetes (18). The authors of the present study therefore hypothesized that SIRT5 may regulate the expression of PDX1 in patients with diabetes. To verify this hypothesis, SIRT5 was overexpressed or knocked down in MIN6 and INS-1 cells and the expression of PDX1 was determined using RT-qPCR and western blotting analyses. The results demonstrated that PDX1 mRNA and protein levels were regulated by SIRT5 (Fig. 3A and B). SIRT5 ectopic expression and inhibition significantly reduced and increased the expression of PDX1, respectively (Fig. 3A and B). To investigate whether the effects of SIRT5 on the expression of PDX1 were dependent on its deacetylase activity, an inactive form of the SIRT5 deacetylase (T69A) was overexpressed. It was demonstrated that ectopic expression of SIRT5 (T69A) had no significant effect on the expression of PDX1 (Fig. 3A and B). Subsequently, a ChIP assay was performed to determine whether SIRT5 regulates the transcription of PDX1 expression through H4K16 deacetylation. The results revealed that occupation of H4K16ac in the PDX1 promoter region was significantly increased following knock down of SIRT5 (Fig. 3C). However, reconstituting the expression of SIRT5R in MIN6 cells could partially offset the effect of SIRT5-depletion (Fig. 3C). Similar results were also observed in INS-1 cells (Fig. 3C). In addition, the expression of PDX1 in plasma samples obtained from 65 patients with diabetes were assessed. The results revealed that SIRT5 expression was negatively associated with PDX1 expression (Table I). Together, these results suggest that the HDAC activity of SIRT5 may regulate the expression of PDX1 via H4K16 deacetylation.

PDX1 is involved in SIRT5-mediated insulin secretion and pancreatic $\beta$-cell proliferation. The results presented thus far have demonstrated that SIRT5 may regulate the transcription of PDX1. Therefore, it was hypothesized that PDX1 may serve as a downstream effector of SIRT5. To verify this hypothesis, the pcDNA3.1-PDX1 plasmid was constructed to increase the expression of PDX1 in MIN6 and INS-1 cells. Western blotting and RT-qPCR analyses were employed to analyze PDX1 protein and mRNA levels, respectively. It was demonstrated that the protein and mRNA levels of PDX1 were significantly increased in MIN6 and INS-1 cells transfected with a PDX1 when compared with controls (Fig. 4A and B). The involvement of PDX1 in SIRT5-mediated insulin secretion and pancreatic $\beta$-cell proliferation was then determined. It was demonstrated that the simultaneous overexpression of SIRT5 and PDX1 offset the effect of SIRT5 overexpression in INS-1 and MIN6 cells (Fig. 4C). When cells were simultaneously 
A

B

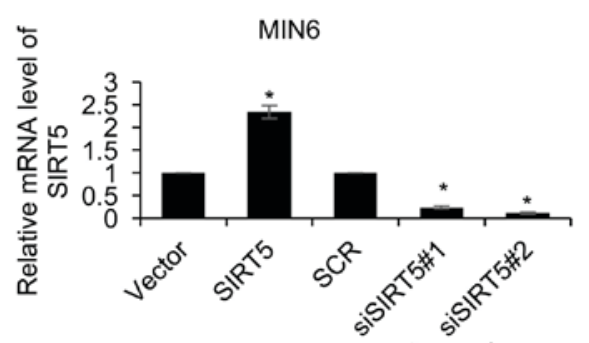

C
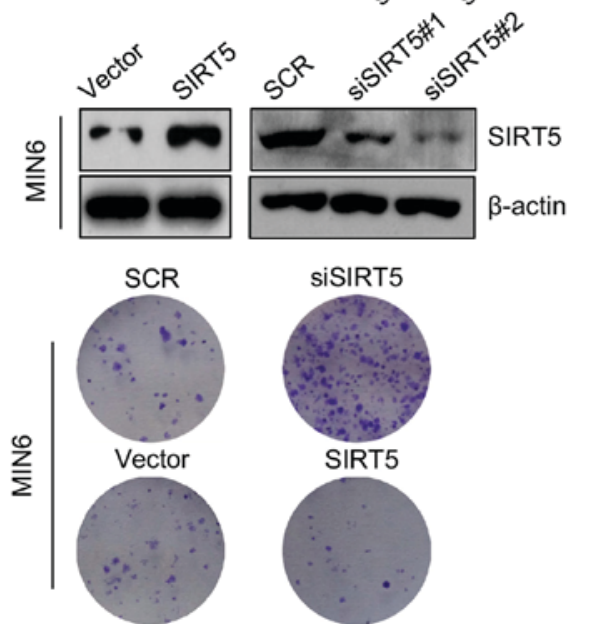

$\mathrm{SCR}$

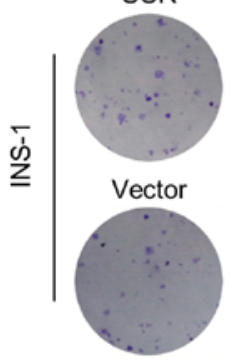

D

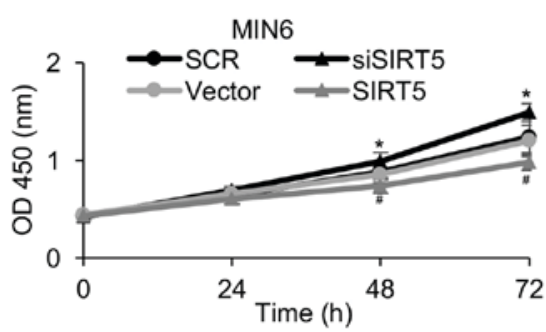

E

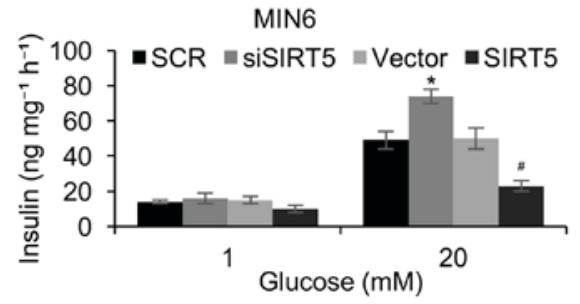

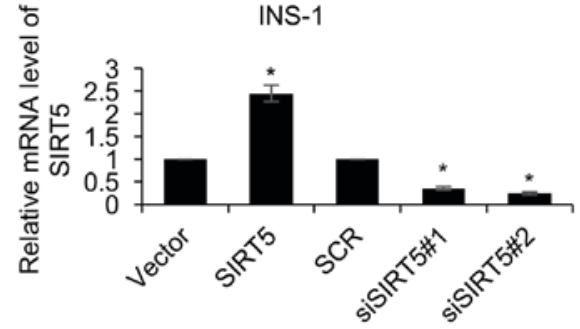

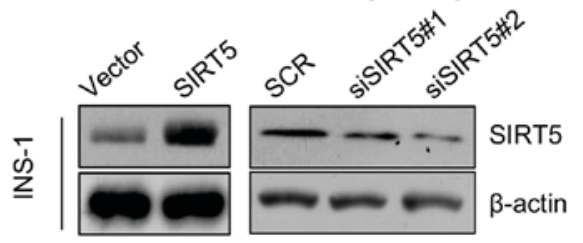

MIN6

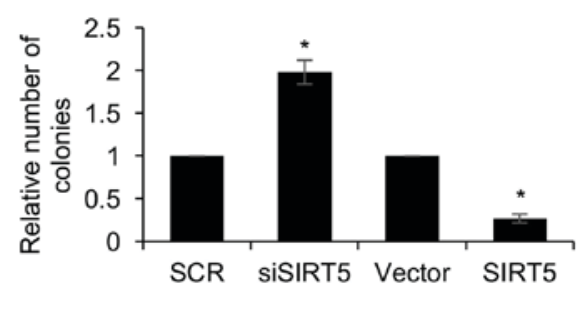

INS-1

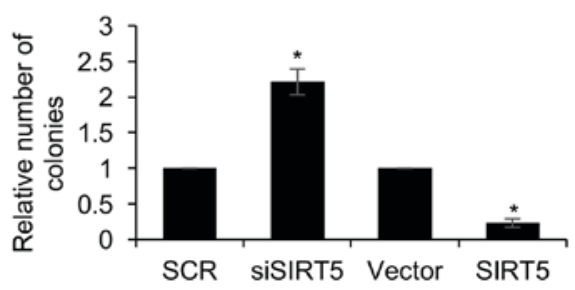

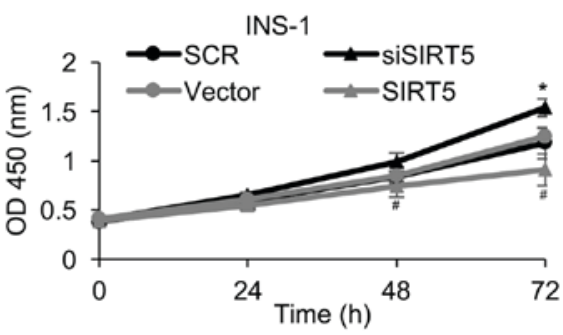

INS-1

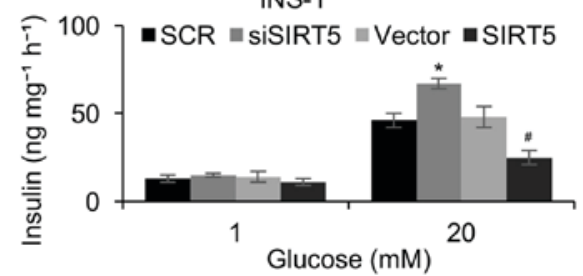

Figure 2. SIRT5 suppresses insulin secretion and pancreatic $\beta$-cell proliferation. MIN6 and INS-1 cells were transfected with an empty plasmid vector, a plasmid vector containing SIRT5, SCR or siSIRT5. At $48 \mathrm{~h}$ following transfection, the (A) mRNA levels of SIRT5 were detected by reverse transcription quantitative polymerase chain reaction analysis and (B) SIRT5 protein levels were detected by western blotting. (C) A colony formation assay and (D) a CCK-8 assay was performed to assess the effect of SIRT5 on cell proliferation. Each experiment was repeated three times. (E) Transfected MIN6 and INS-1 cells were also incubated with $1 \mathrm{mM}$ or $20 \mathrm{mM}$ glucose for $90 \mathrm{~min}$ and the rate of insulin secretion was detected in each group. Each experiment was repeated three times. The results are presented as the mean \pm standard deviation. * $\mathrm{P}<0.05$ vs. SCR and ${ }^{\#} \mathrm{P}<0.05$ vs. vector. SIRT5, sirtuin 5; Vector, pcDNA3.1 plasmid vector; SCR, scrambled small interfering RNA; siSIRT5, small interfering RNA targeting sirtuin 5; OD, optical density.

overexpressed with SIRT5 and PDX1, the glucose-stimulated secretion of insulin was increased compared with the cells overexpressing SIRT5 (Fig. 4C). Similarly, the CCK-8 assay results demonstrated that combined SIRT5 and PDX1 overexpression significantly reduced the effect of the SIRT5 overexpression on cell proliferation (Fig. 4D). These results 
A

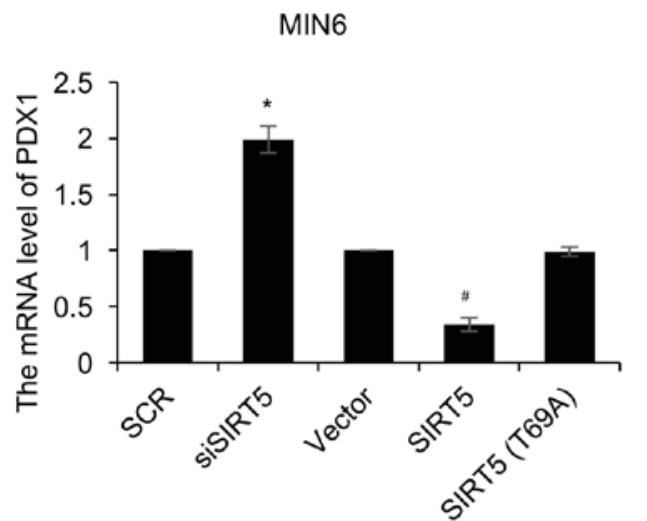

B

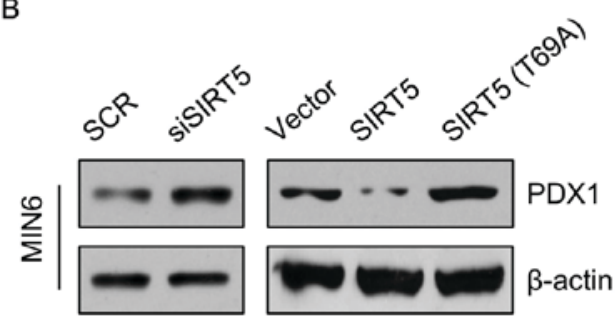

C

MIN6

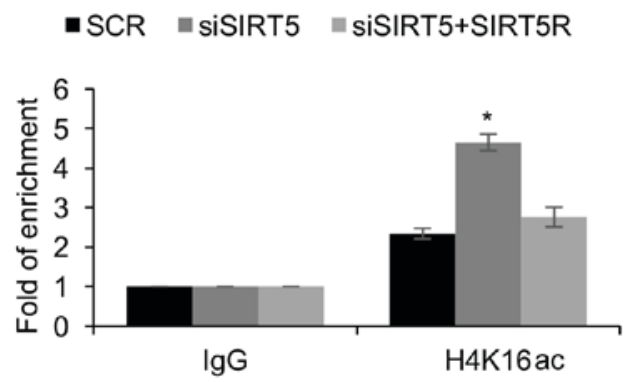

INS-1
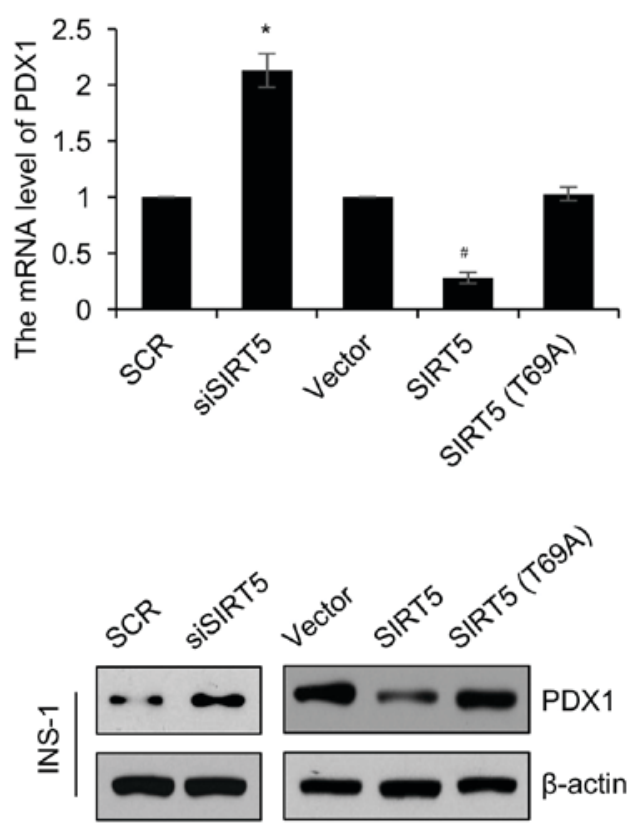

INS-1

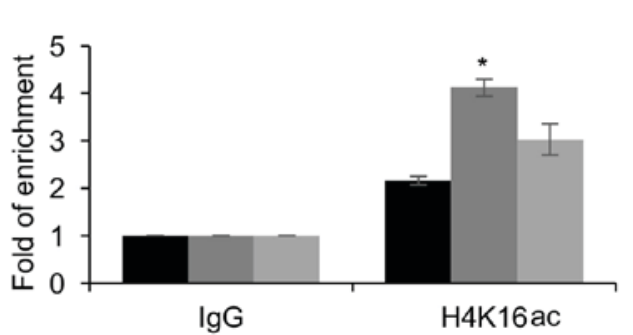

Figure 3. PDX1 is transcriptionally regulated by SIRT5. MIN6 and INS-1 cells were an empty plasmid vector, a plasmid vector containing SIRT5, SCR, siSIRT5 or SIRT5 (T69A). Following $48 \mathrm{~h}$ transfection, the (A) mRNA and (B) protein levels of PDX1 was detected using reverse transcription quantitative polymerase chain reaction and western blotting analyses, respectively. (C) SIRT5 was knocked down in treated MIN6 and INS-1 cells, and anti-H4K16ac antibodies were used to perform qChIP assays. Each experiment was repeated three times. The results are presented as the mean \pm standard deviation. ${ }^{*} \mathrm{P}<0.05$ vs. SCR and ${ }^{\#} \mathrm{P}<0.05$ vs. vector. SIRT5, sirtuin 5; Vector, pcDNA3.1 plasmid vector; SCR, scrambled small interfering RNA; siSIRT5, small interfering RNA targeting sirtuin 5; PDX1, pancreatic and duodenal homeobox 1; qChIP, quantitative chromatin immunoprecipitation.

indicate that PDX1 may function as a downstream target of SIRT5 and mediate pancreatic $\beta$-cell proliferation and insulin secretion.

VPA increases PDX1 expression and insulin secretion. Due to the HDAC activity of SIRT, the effect of VPA treatment (a HDAC inhibitor) on pancreatic $\beta$-cells was assessed. MIN6 and INS-1 cells incubated with $0.1,0.5$ and $1 \mathrm{mM}$ VPA for $48 \mathrm{~h}$ exhibited a significant increase in the expression of PDX1 (Fig. 5A). In addition, treatment with increasing concentrations of VPA was associated with a dose-dependent increase in insulin secretion following exposure to glucose and SIRT5 overexpression decreased insulin secretion compared with the control group (Fig. 5B). The results therefore suggest that VPA may be a novel therapy for $\mathrm{T} 2 \mathrm{D}$, which is consistent with the results of previous studies $(19,20)$.

\section{Discussion}

The dysfunction of $\beta$-cells is a primary cause of human T2D and results from the abnormal expression of specific genes involved in regulating metabolic signaling pathways $(21,22)$. Chronically high carbohydrate diets may also contribute to $\beta$-cell dysfunction (21). SIRT5 has been demonstrated to serve key roles in various metabolic processes $(10,23,24)$, including deacetylation and desuccinylation of CPS1 by SIRT5, resulting in its increased enzymatic activity, thereby promoting the urea cycle (25). However, the role of SIRT5 in T2D has not yet been elucidated.

The present study demonstrated that SIRT5 was upregulated in patients with T2D and that the expression of SIRT5 was associated with high blood glucose levels. It was also revealed that the expression of SIRT5 was associated with 
A

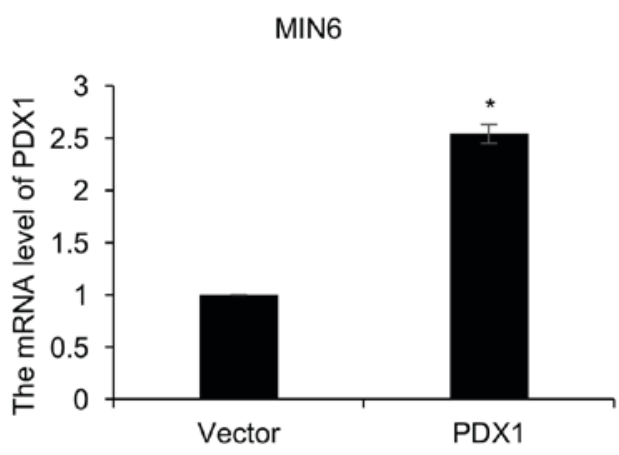

B

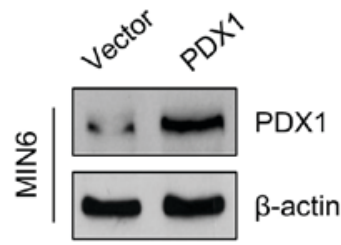

C

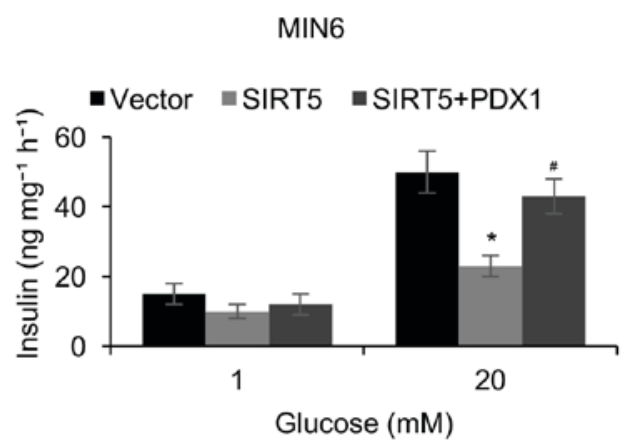

D

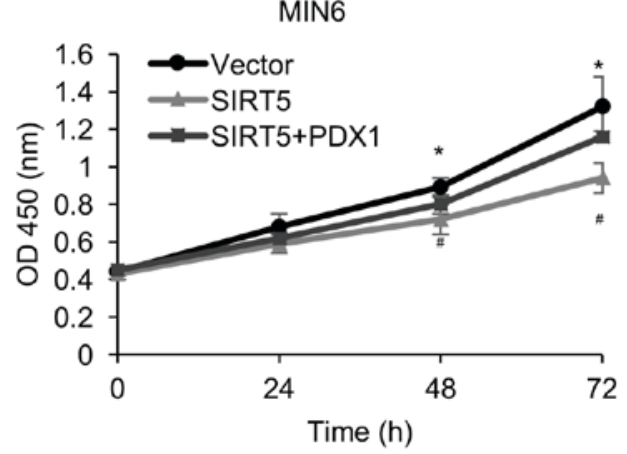

INS-1
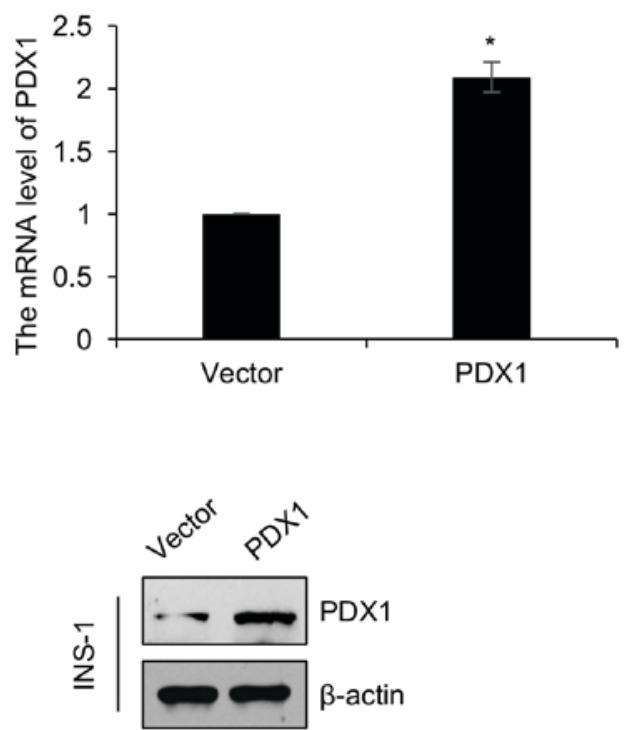

INS-1

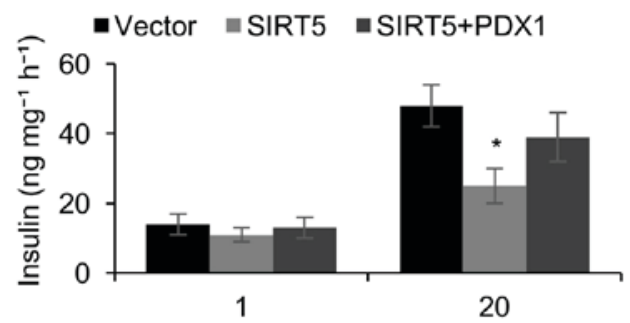

Glucose (mM)

INS-1

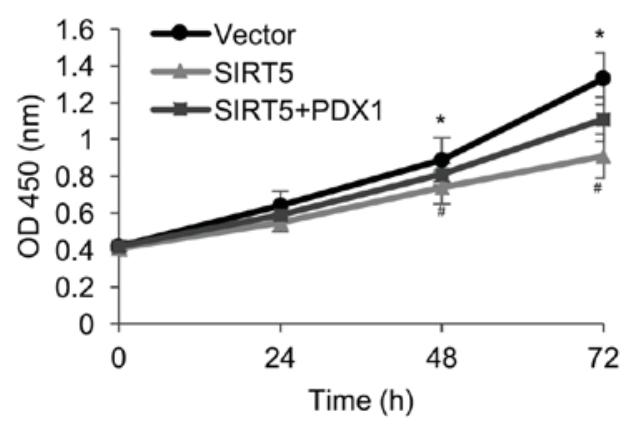

Figure 4. PDX1 is involved in SIRT5-mediated pancreatic $\beta$-cell proliferation and insulin secretion. PDX1 was overexpressed in MIN6 and INS-1 cells. Following $48 \mathrm{~h}$ transfection, PDX1 (A) mRNA and (B) protein levels were assessed using reverse transcription quantitative polymerase chain reaction and western blotting, respectively. MIN6 and INS-1 cells were transfected with an empty plasmid vector, a SIRT5 expression plasmid or with expression plasmids containing SIRT5 and PDX1 simultaneously. Following $48 \mathrm{~h}$ transfection, cells were incubated with 1 or $20 \mathrm{mM}$ glucose for $90 \mathrm{~min}$ and (C) the secretion of insulin was assessed in each group. (D) A CCK-8 assay was performed on the same groups of MIN6 and INS-1 cells, to assess the effects of SIRT5 on cell proliferation. Each experiment was repeated three times. The results are presented as the mean \pm standard deviation. ${ }^{*} \mathrm{P}<0.05$ vs. Vector and ${ }^{\#} \mathrm{P}<0.05$ vs. SIRT5. PDX1, pancreatic and duodenal homeobox 1; SIRT5, sirtuin 5; OD, optical density; Vector, pcDNA3.1 plasmid vector.

patient age. This may be due to alterations in the expression levels of specific proteins as the body ages, such as SIRT1 (26), resulting in a high expression of SIRT5. To investigate the effect of SIRT5 in T2D in the present study, two pancreatic $\beta$-cell lines (MIN6 and INS-1) were utilized. Functional experiments, including colony formation and CCK-8 assays, were performed. The results suggested that SIRT5 inhibition facilitated pancreatic $\beta$-cell proliferation and insulin secretion.
A previous study demonstrated that SIRT5 facilitates cancer cell growth and drug resistance in non-small cell lung cancer (27). This contradicts the results of the present study; however, it should be considered that diabetes and cancer are two different diseases. The present study hypothesized that there are also many factors that may contribute to the different functions of SIRT5, including cell microenvironment, metabolism and oncogene activation. 
A
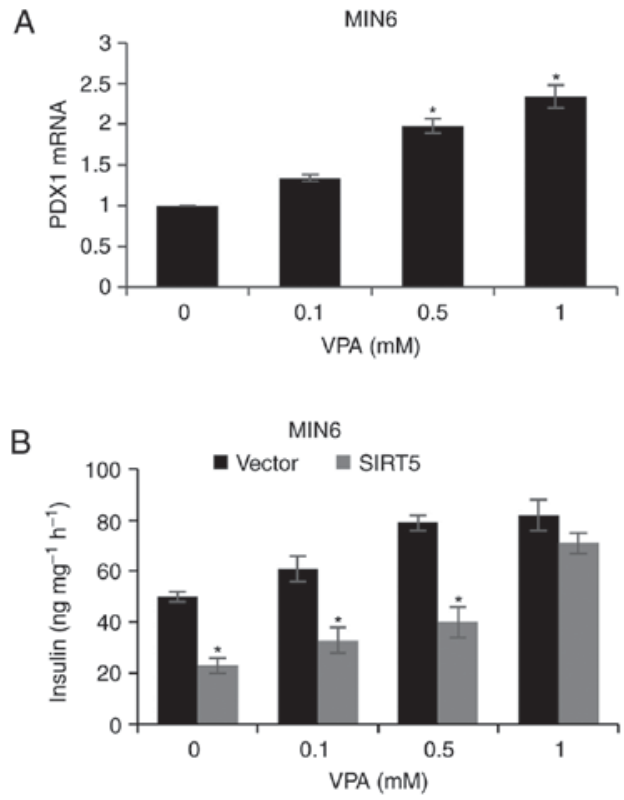

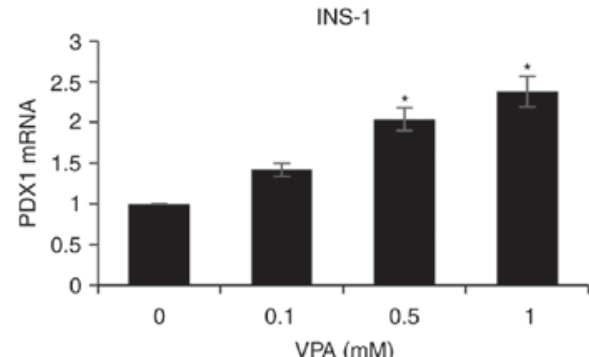

INS-1

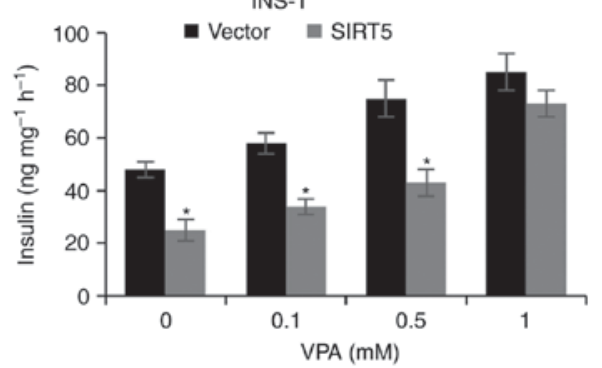

Figure 5. VPA elevates PDX1 expression and insulin secretion. (A) MIN6 and INS-1 cells were treated with increasing concentrations of VPA. Following 48 h treatment, PDX1 mRNA levels were analyzed by reverse transcription quantitative polymerase chain reaction analysis ( ${ }^{*} \mathrm{P}<0.05 \mathrm{vs}$. 0 mM VPA). (B) SIRT5 was knocked down in MIN6 and INS-1 cells prior to treatment with $20 \mathrm{mM}$ glucose and increasing concentrations of VPA for $48 \mathrm{~h}$, and the level of insulin secretion was subsequently determined. Each experiment was repeated three times. The results are presented as the mean \pm standard deviation $\left({ }^{*} \mathrm{P}<0.05 \mathrm{vs}\right.$. Vector). VPA, valproic acid; PDX1, pancreatic and duodenal homeobox 1; SIRT5, sirtuin 5; Vector, pcDNA3.1 plasmid vector.

PDX1 is the earliest tissue-selective transcription factor expressed in the developing primordium, and is essential to formation of all pancreatic cell types and the activity of adult islet $\beta$ cells (28). Thus, mice and humans that completely lack Pdx1 function are apancreatic, while haploinsufficiency primarily affects islet $\beta$ cells following birth (28). Moreover, $\beta$ cell-specific inactivation of $\mathrm{Pdx} 1$ in the adult mouse causes severe hyperglycemia and loss of cell identity, with these cells transdifferentiating to an islet $\alpha$-like cell capable of secreting the glucagon hormone (18). Previous studies have demonstrated that PDX1 serves a primary role in diabetes and that the downregulation of PDX1 expression aggravates diabetes mellitus $(29,30)$. The results of the present study demonstrated that SIRT5 may regulate the transcription of PDX1 through its deacetylase activity. Despite SIRT5 only possessing weak deacetylase activity, it was demonstrated that PDX1 is negatively regulated by SIRT5. A previous study suggested that SIRT5 may function through its de-succinylase activity (31). However, the role of succinylation in cancer and metabolic diseases remains unknown. It is therefore important to assess alterations in the level of specific histone or protein succinylation in patients with diabetes mellitus using mass spectrometry in future studies.

Previous studies have demonstrated that the HDAC inhibitor, VPA, increases insulin secretion in vivo $(19,20)$. Consistent with these results, the present study revealed that VPA facilitated the secretion of insulin in two pancreatic $\beta$-cell lines. In addition, it was determined that VPA may promote insulin secretion by enhancing the expression of PDX1.

In conclusion, the current study investigated the function of SIRT5 in T2D and the mechanisms underlying it role in insulin secretion and pancreatic $\beta$-cell proliferation. The results revealed that SIRT5 may be associated with the pathogenesis of T2D and may serve as a novel biomarker for the diagnosis of T2D.

\section{Acknowledgements}

Not applicable.

\section{Funding}

No funding was received.

\section{Availability of data and materials}

The datasets used and/or analyzed during the current study are available from the corresponding author on reasonable request

\section{Authors' contributions}

YM and XF conceived and designed the study, analyzed the data, and wrote the paper. YM constructed the expression plasmids, prepared the proteins, and performed experiments. All authors have read and approved this manuscript.

\section{Ethics approval and consent to participate}

The present study was approved by the Ethics Committee of Taizhou People's Hospital (Taizhou, China) and written informed consent was provided from patients and healthy volunteers prior to enrolment.

\section{Consent for publication}

Not applicable. 


\section{Competing interests}

The authors declare that they have no competing interests.

\section{References}

1. Matzinger M, Fischhuber K and Heiss EH: Activation of Nrf2 signaling by natural products-can it alleviate diabetes? Biotechnol Adv: Dec 28, 2017 (Epub ahead of print).

2. Stoffers DA: The development of beta-cell mass: Recent progress and potential role of GLP-1. Horm Metab Res 36: 811-821, 2004

3. Tourrel C, Bailbe D, Lacorne M, Meile MJ, Kergoat M and Portha B: Persistent improvement of type 2 diabetes in the Goto-Kakizaki rat model by expansion of the beta-cell mass during the prediabetic period with glucagon-like peptide-1 or exendin-4. Diabetes 51: 1443-1452, 2002.

4. Sakuraba H, Mizukami H, Yagihashi N, Wada R, Hanyu C and Yagihashi S: Reduced beta-cell mass and expression of oxidative stress-related DNA damage in the islet of Japanese Type II diabetic patients. Diabetologia 45: 85-96, 2002.

5. Marchetti P, Del Guerra S, Marselli L, Lupi R, Masini M, Pollera M, Bugliani M, Boggi U, Vistoli F, Mosca F and Del Prato S: Pancreatic islets from type 2 diabetic patients have functional defects and increased apoptosis that are ameliorated by metformin. J Clin Endocrinol Metab 89: 5535-5541, 2004

6. Cozar-Castellano I, Fiaschi-Taesch N, Bigatel TA, Takane KK, Garcia-Ocaña A, Vasavada R and Stewart AF: Molecular control of cell cycle progression in the pancreatic beta-cell. Endocr Rev 27: 356-370, 2006.

7. Hirschey MD: Old enzymes, new tricks: Sirtuins are NAD(+)-dependent de-acylases. Cell Metab 14: 718-719, 2011.

8. Colak G, Xie Z, Zhu AY, Dai L, Lu Z, Zhang Y, Wan X, Chen Y, Cha $\mathrm{YH}$, Lin $\mathrm{H}$, et al: Identification of lysine succinylation substrates and the succinylation regulatory enzyme CobB in Escherichia coli. Mol Cell Proteomics 12: 3509-3520, 2013.

9. Park J, Chen Y, Tishkoff DX, Peng C, Tan M, Dai L, Xie Z, Zhang Y, Zwaans BM, Skinner ME, et al: SIRT5-mediated lysine desuccinylation impacts diverse metabolic pathways. Mol Cell 50: 919-930, 2013

10. Rardin MJ, He W, Nishida Y, Newman JC, Carrico C, Danielson SR, Guo A, Gut P, Sahu AK, Li B, et al: SIRT5 regulates the mitochondrial lysine succinylome and metabolic networks. Cell Metab 18: 920-933, 2013.

11. Weinert BT, Scholz C, Wagner SA, Iesmantavicius V, Su D, Daniel JA and Choudhary C: Lysine succinylation is a frequently occurring modification in prokaryotes and eukaryotes and extensively overlaps with acetylation. Cell Rep 4: 842-851, 2013.

12. Zhang Z, Tan M, Xie Z, Dai L, Chen Y and Zhao Y: Identification of lysine succinylation as a new post-translational modification. Nat Chem Biol 7: 58-63, 2011.

13. Hirschey MD and Zhao Y: Metabolic regulation by lysine malonylation, succinylation, and glutarylation. Mol Cell Proteomics 14: 2308-2315, 2015 .
14. Tan M, Peng C, Anderson KA, Chhoy P, Xie Z, Dai L, Park J, Chen Y, Huang $\mathrm{H}$, Zhang Y, et al: Lysine glutarylation is a protein posttranslational modification regulated by SIRT5. Cell Metab 19: 605-617, 2014.

15. Xie Z, Dai J, Dai L, Tan M, Cheng Z, Wu Y, Boeke JD and Zhao Y: Lysine succinylation and lysine malonylation in histones. Mol Cell Proteomics 11: 100-107, 2012.

16. American Diabetes Association: Diagnosis and classification of diabetes mellitus. Diabetes Care 35 (Suppl 1): S64-S71, 2012.

17. Livak KJ and Schmittgen TD: Analysis of relative gene expression data using real-time quantitative PCR and the 2(-Delta Delta C(T)) method. Methods 25: 402-408, 2001.

18. Fujimoto $\mathrm{K}$ and Polonsky KS: Pdx 1 and other factors that regulate pancreatic beta-cell survival. Diabetes Obes Metab 11 (Suppl 4): S30-S37, 2009.

19. Manaka K, Nakata M, Shimomura K, Rita RS, Maejima Y, Yoshida M, Dezaki K, Kakei M and Yada T: Chronic exposure to valproic acid promotes insulin release, reduces KATP channel current and does not affect $\mathrm{Ca}(2+)$ signaling in mouse islets. J Physiol Sci 64: 77-83, 2014.

20. Axelsson AS, Mahdi T, Nenonen HA, Singh T, Hänzelmann S, Wendt A, Bagge A, Reinbothe TM, Millstein J, Yang X, et al: Sox 5 regulates beta-cell phenotype and is reduced in type 2 diabetes. Nat Commun 8: 15652, 2017.

21. Weir GC and Bonner-Weir S: Five stages of evolving beta-cell dysfunction during progression to diabetes. Diabetes 53 (Suppl 3): S16-S21, 2004

22. Ashcroft FM and Rorsman P: Diabetes mellitus and the $\beta$ cell: The last ten years. Cell 148: 1160-1171, 2012.

23. Kumar S and Lombard DB: Mitochondrial sirtuins and their relationships with metabolic disease and cancer. Antioxid Redox Signal 22: 1060-1077, 2015.

24. Yu J, Sadhukhan S, Noriega LG, Moullan N, He B, Weiss RS, Lin H, Schoonjans K and Auwerx J: Metabolic characterization of a Sirt5 deficient mouse model. Sci Rep 3: 2806, 2013.

25. Nakagawa T, Lomb DJ, Haigis MC and Guarente L: SIRT5 Deacetylates carbamoyl phosphate synthetase 1 and regulates the urea cycle. Cell 137: 560-570, 2009.

26. Qadir MI and Anwar S: Sirtuins in brain aging and neurological disorders. Crit Rev Eukaryot Gene Expr 27: 321-329, 2017.

27. Lu W, Zuo Y, Feng Y and Zhang M: SIRT5 facilitates cancer cell growth and drug resistance in non-small cell lung cancer. Tumour Biol 35: 10699-10705, 2014.

28. Gannon M, Gamer LW and Wright CV: Regulatory regions driving developmental and tissue-specific expression of the essential pancreatic gene pdx1. Dev Biol 238: 185-201, 2001.

29. Spaeth JM, Gupte M, Perelis M, Yang YP, Cyphert H, Guo S, Liu JH, Guo M, Bass J, Magnuson MA, et al: Defining a novel role for the Pdx 1 transcription factor in islet $\beta$-cell maturation and proliferation during weaning. Diabetes 66: 2830-2839, 2017.

30. Fujitani Y: Transcriptional regulation of pancreas development and $\beta$-cell function [Review]. Endocr J 64: 477-486, 2017.

31. Du J, Zhou Y, Su X, Yu JJ, Khan S, Jiang H, Kim J, Woo J, Kim JH, Choi BH, et al: Sirt5 is a NAD-dependent protein lysine demalonylase and desuccinylase. Science 334: 806-809, 2011. 